\title{
Alterations in Glucose Turnover Following Single Intravenous Injections of Epinephrine and Glucagon in Dogs
}

\author{
By Shafeer S. Sanbar
}

The technique of priming injection-continuous infusion of tracer glucose-U.C ${ }^{14}$ was employed in 7 anesthetized dogs, each serving as its own control, to determine the immediate changes in rates of appearance ( $\mathrm{Ra}$ ) and disappearance (Rd) of plasma glucose following single intravenous injections of epinephrine (10 $\mu \mathrm{g}$. per $\mathrm{Kg}$.) and glucagon ( $1 \mu \mathrm{g}$. per Kg.). Mean maximal hyperglycemia induced by the hormones was comparable in magnitude, but it was achieved in 4 minutes after epinephrine injection compared with 8 to 10 minutes after glucagon. Furthermore, Ra was increased 5- to 7-fold during the first 4 minutes following epinephrine injection and sub- sequently fell to below control levels for about 30 minutes. In contrast, following glucagon, Ra was increased 3- to 4-fold during the first 8 minutes after injection with subsequent return to control levels. After both epinephrine and glucagon, $\mathrm{Rd}$ increased 1.5- to 2-fold during the first 10 minutes; it returned to control levels 10 minutes after epinephrine and 30 minutes after glucagon. These data indicate that the increment in plasma glucose results from enhanced hepatic glycogenolysis. However, contrary to the suggestion of others, the effect of epinephrine on the liver does not appear to be mediated via glucagon release. (Metabolism 17: No. 7, July, 631-637, 1968)

$\mathbf{I}^{\mathrm{T}}$

T IS WELL ESTABLISHED that glucagon induces hyperglycemia by directly enhancing hepatic glycogenolysis and gluconeogenesis. ${ }^{2}$ In contrast, it has been shown both in vivo and in vitro that, except at near lethal concentrations, epinephrine induces hyperglycemia by an indirect action of the hormone on the liver. ${ }^{3-\overline{5}}$ In studies with rats $^{2}$ and dogs, ${ }^{4}$ infusion of $0.3 \mu \mathrm{g}$. epinephrine per $\mathrm{Kg}$. per minute into a peripheral vein enchances hepatic glycogenolysis and increases blood lactate concentration. On the other hand, the same authors have reported that infusion of an identical dose of epinephrine into the portal vein of the animals produces no effect on either hepatic glycogenolysis or blood lactate concentration. Studies using isolated perfused rat liver have demonstrated also that two additions of $3 \mu \mathrm{g}$. of epinephrine to the perfusate do not alter hepatic glycogen content or glucose concentration in the

From the Department of Internal Medicine, Unitersity of Michigan, Ann Arbor, Michigan.

This work was supported in part by a grant from the Michigan Heart Foundation and was completed during the tenure of an Advance Research Fellowship of the American Heart Association.

Presented in part at the Twenty-fourth Annual Meeting of the Midwestern Section of the American Federation for Clinical Research, November 3, 1966, Chicago, Ill.; results have been published in abstract form (1).

Received for publication September 25, 1967.

Shafeek S. Sanbar, M.D., Ph.D.: Assistant Professor, Department of Internal Medicine, University of Michigan, Ann Arbor, Mich. 
perfusate; only at epinephrine concentrations that are nonphysiologic, in excess of $100 \mu \mathrm{g}$. per liter, could a glycogenolytic effect on the liver be demonstrated. ${ }^{2,5}$ Indeed, Ezdinli and Sokal ${ }^{4}$ concluded from their comparative studies in dogs and rats that "only glucagon can serve as a physiologic hepatic glycogenolytic agent, and that the effect of moderate doses of epinephrine (10 $\mu$ g. per Kg.) on the liver is an indirect one, possibly mediated through stimulation of glucagon secretion."

It was believed by this author that if the effect of epinephrine on hepatic glycogenolysis were mediated through glucagon, one would expect following the administration of these hormones in dose levels which elicit comparable maximal hyperglycemia that glucose turnover would be altered either in an identical manner or that the effect of glucagon on the liver would precede that of epinephrine. This study in anesthetized dogs compares the immediate effects of single intravenous injections of epinephrine and glucagon on rates of glucose production (primarily hepatic output) ${ }^{6}$ and utilization (tissue uptake), using the technic of priming injection-continuous infusion of tracer glucose-U-C ${ }^{14} \cdot{ }^{7}$ The results show that with a comparable hyperglycemic response, the maximal increase in plasma concentration and rate of appearance of glucose induced by epinephrine precede in time those produced by glucagon. Contrary to the suggestion of others, therefore, the hepatic glycogenolytic effect of epinephrine is apparently not mediated via glucagon. Interestingly enough, the results also show that the rates of disappearance of glucose from circulation are increased to almost the same degree immediately following the injection of epinephrine and glucagon. This indicates that the increase in plasma glucose induced by single injections of these hormones is totally due to the increase in hepatic glucose output. A possible mechanism by which epinephrine enhances hepatic glycogenolysis is discussed.

\section{Materials and Methods}

Scven male and female mongrel dogs weighing 12 to $17 \mathrm{Kg}$. were used in this study. After an overnight fast, the dogs were anesthetized with pentobarbital sodium ( $30 \mathrm{mg}$./ $/ \mathrm{Kg}$. body weight). A medium-size catheter (Intracath, C. R. Bard, Inc., Murray Hill, N. J.) was inserted in the cephalic vein for intravenous infusions and a polyethylene tube in the femoral artery for repeated blood sampling. The experimental procedures lasted 3 to $4 \frac{11}{2}$ hours, and each dog served as its own control.

The technic of priming injection-continuous intravenous infusion of tracer glucose-U$\mathrm{C}^{14} 7$ was utilized to determine the immediate changes in rates of appearance ( $\mathrm{Ra}$ ) and disappearance ( $\mathrm{Rd}$ ) of glucose following intravenous administration of epinephrine and glucagon. The dose of tracer, calculations of $\mathrm{Ra}$ and $\mathrm{Rd}$ and laboratory methods were identical to those reported recently in this Journal. 8

After a control period of 2 hours, 3 dogs received a single injection of epinephrine (10 $\mu \mathrm{g}$. per Kg.) over a period of 2 to 3 seconds via a 22-gauge needle inserted in the saphenous vein. One hour after epinephrine administration, each dog received also intravenously a single injection of glucagon $(1 \mu \mathrm{g}$. per $\mathrm{Kg}$.) in a similar manner. Three other dogs received only a single intravenous injection of glucagon after the 2-hour control period. The last dog received also intravenously both epinephrine and glucagon simultaneously. The dose levels of $10 \mu \mathrm{g}$. epinephrine and $1 \mu \mathrm{g}$. glucagon per $\mathrm{Kg}$. body weight were selected because preliminary studies revealed that in the same dog these dose levels produced a comparable maximal hyperglycemic response. 


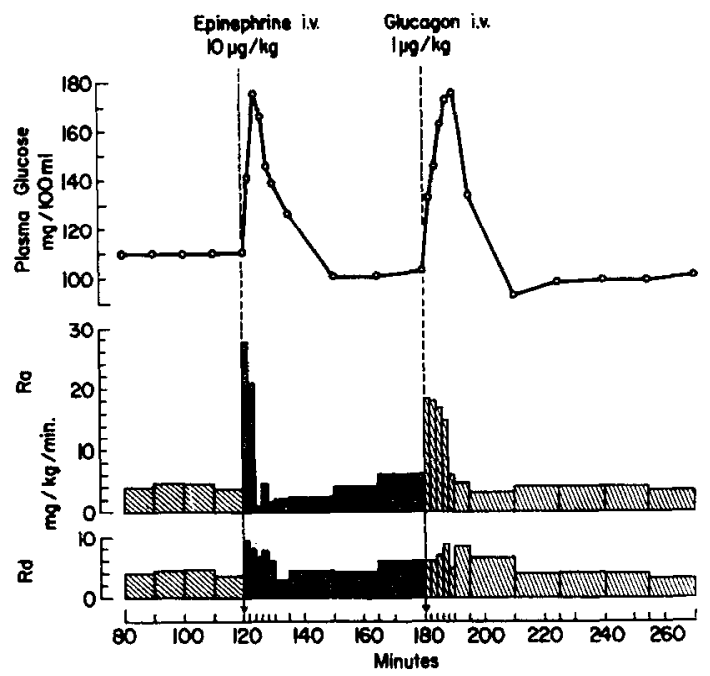

Fig. 1.--Mean changes in plasma glucose concentration and rates of appearance $(\mathrm{Ra})$ and disappearance $(\mathrm{Rd})$ of plasma glucose in 3 dogs induced by intravenous injections of epinephrine followed by glucagon.

Glucose-U-C ${ }^{14}$ was infused into the cephalic vein, while arterial blood samples were obtained at 2-, 5- or 15-minute intervals throughout the procedures.

\section{Results}

The single intravenous injection of epinephrine produced uniformly the following immcdiate mean alterations in glucose metabolism in 3 dogs (Fig. 1). Plasma glucose concentration was consistently increased with peak hyperglycemia occurring 4 minutes after injection; plasma glucose concentration returned to control levels 30 minutes after injection. During the control period, the rates of glucose appearance (Ra) and disappearance (Rd) were equal averaging approximately $4 \mathrm{mg}$. per $\mathrm{Kg}$. per minute. During the first 4 minutes after epinephrine injection, $\mathrm{Ra}$ was increased by 5 - to 7 -fold. Ra fell subsequently to below control levels averaging about $2 \mathrm{mg}$. per $\mathrm{Kg}$. per minute between 4 and 30 minutes, and finally it returned to control levels. Rd was also increased by 1.5- to 2-fold of control level during the first ten minutes after injection, with a subsequent return to control level.

In contrast with epinephrine, an intravenous injection of glucagon in 6 dogs produced a delayed hyperglycemic response (Figs. 1 and 2). Although the increase in plasma glucose concentration was comparable to that following epinephrine injection, the peak hyperglycemia occurred 8 to 10 minutes after glucagon injection. Plasma glucose specific activity was decreased, and Ra was increased by 3 - to 4 -fold above control level during the first 8 minutes after glucagon; Ra returned to control the levels subsequently. Also after glucagon, there was a 1.5 to 2 fold increase in Rd which lasted about 30 minutes; Rd returned to control level subsequently.

Finally, the single experiment giving an injection of both epinephrine and glucagon (Fig. 3) produced an additive hyperglycemic response and a 10fold increase in Ra above control during the first 6 minutes after injection and 4-fold during the next 4 minutes. Also, Rd was increased by 4 -fold during 
Fig. 2.-Mean changes in plasma glucose concentration, glucose specific activity, Ra and Rd in 3 dogs induced by intravenous injection of glucagon.

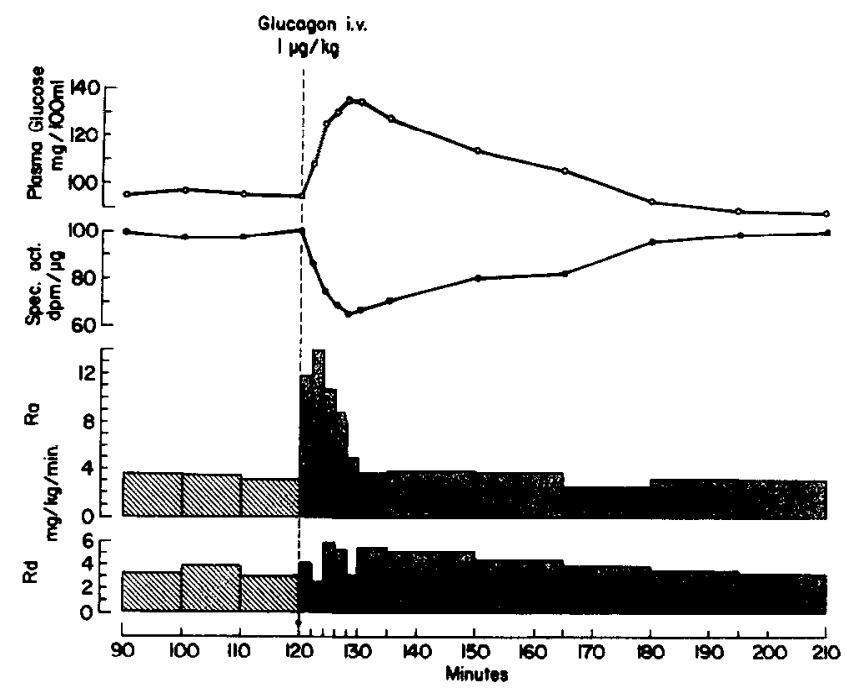

Fig. 3.-Changes in plasma glucose concentration, $\mathrm{Ra}$ and $\mathrm{Rd}$ induced by simultaneous intravenous injection of both epinephrine and glucagon in a dog.

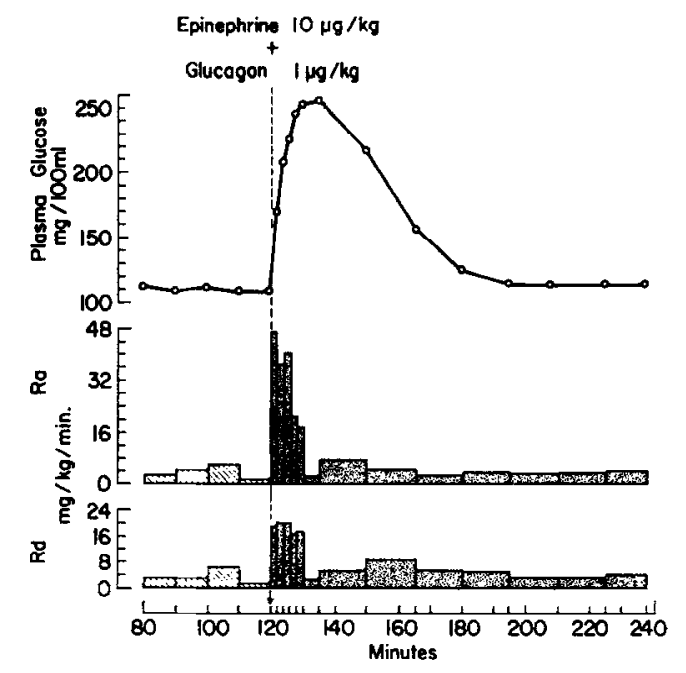

the first 10 minutes after injection. All values returned to control levels about 1 hour after injection of the hormones.

\section{Discussion}

These studies in dogs have demonstrated the immediate alterations in glucose turnover following single injections of epinephrine and glucagon. In the same dog epinephrine is approximately 10 times less potent than glucagon on a weight basis as a hepatic glycogenolytic agent. The peak hyperglycemia following epinephrine precedes that of glucagon. Epinephrine is in fact about twice as fast as glucagon in producing maximal hyperglycemia. Also, as with the change in plasma glucose, the rate of appearance or hepatic output of glucose also responds faster to epinephrine injection than to glucagon. The maximal increase occurs within the first 4 minutes after epinephrine, while 
glucagon induces a comparatively smaller though longer lasting increase in $\mathrm{Ra}$. The rate of disappearance or tissue uptake of glucose is increased to almost the same degree by both epinephrine and glucagon. It is concluded, contrary to the suggestion of others, ${ }^{4}$ that both epinephrine and glucagon are physiologic hepatic glycogenolytic agents and that the effect of epinephrine on the liver is apparently not mediated through glucagon. Since the hepatic effect of epinephrine is not mediated through glucagon, what then are the possible alternatives for the mechanism of action of this hormone on the liver? The following discussion outlines the current status of knowledge concerning this complex phenomenon which appears to be made up of several components.

Epinephrine Hyperglycemia Results from Enhanced Hepatic Glucose Output

Single injections of $1-10 \mu \mathrm{g}$. of epinephrine per $\mathrm{Kg}$. body weight in dogs, cats and rabbits have been shown to produce peak hyperglycemia between 3 and 5 minutes after injection, the maximum increase in blood glucose being 40 to $70 \mathrm{mg}$. per $100 \mathrm{ml}$. above control values. ${ }^{9-11}$ The data presented here are in keeping with these findings. The hyperglycemia induced by epinephrine has also been shown to be associated with enhanced hepatic glucose output. ${ }^{12-13}$ Since it has been established in this study that the glucose uptake by tissues is increased immediately following epinephrine injection, it follows that the hyperglycemia induced by the hormone is totally accounted for by the enhanced hepatic glucose output.

\section{Direct Action of Epinephrine on the Liver}

It is well known that epinephrine is capable of directly stimulating hepatic glycogenolysis. ${ }^{14}$ As pointed out by Sokal et al., ${ }^{5}$ however, the direct action of epinephrine on the liver requires pharmacological doses of the hormone for even minimal glycogenolytic effect. It must also be emphasized that the direct action of epinephrine on the liver is not an immediate one as seen in vivo but is delayed. Using liver slices of rabbits, Sutherland and Cori ${ }^{15}$ have shown that epinephrine in concentrations of about $7 \mu \mathrm{g}$. per $100 \mathrm{ml}$. of incubate does not enhance initially glucose production by the slices but does so 30 to 40 minutes after incubation. Similarly, using isolated perfused liver of rabbits, Lundsgaard, Neilsen and Orskov ${ }^{16,17}$ reported no increase in glucose concentration in the perfusate during the first 10 minutes after the start of an epinephrine infusion ( $1 \mu \mathrm{g}$. per min.); glucose concentration in the perfusate was increased subsequently. In contrast with in vitro findings, this study has demonstrated that the effect of epinephrine on $\mathrm{Ra}$ is immediate. This makes it unlikely that the hepatic action of moderate doses of epinephrine, as used in this study, is a direct one.

\section{Indirect Action of Epinephrine on the Liver}

In 1949, Sherlock ${ }^{3}$ first demonstrated in rats that intraportal infusion of epinephrine in dose levels of $0.3 \mu \mathrm{g}$. per $\mathrm{Kg}$. per min. produces neither hepatic glycogenolysis nor hyperglycemia during the first 15 minutes after starting the infusion. On the other hand, administration of the same dose of epinephrine via a peripheral vein induces both hepatic glycogenolysis and hyper- 
glycemia. These findings have been confirmed recently in dogs. ${ }^{4}$ It is obvious from these data that at least part of the hepatic glycogenolytic effect of epinephrine is an indirect one.

Central Nervous System Component. Leimdorfer et al. ${ }^{18}$ have shown that injection of epinephrine into the subarachnoid space of cats causes a high and sustained elevation in blood glucose concentration. Rosenberg and DiStefano $^{11}$ have reported also in cats that at least half of the hyperglycemic effect of intravenously injected epinephrine results from activation of a "hyperglycemic center" which is located in the floor of the fourth ventricle. They also point out that this epinephrine-responsive center correlates well with the hyperglycemic area which Bernard described in 1849 by piquire of the floor of the fourth ventricle. The mechanism by which the message is transmitted from the "hyperglycemic center" to the liver, however, is not clear. It could theoretically be mediated through a hormone or possibly transmitted by autonomic nerves either directly to the liver or indirectly via the pancreas. ${ }^{11,19}$

Pancreatic Component. Presently, it is well-established that epinephrine is capable of inhibiting insulin secretion. ${ }^{20-22}$ Since it is known that acute insulin deprivation produces an immediate increase in hepatic glucose output, ${ }^{\mathbf{2 3 . 2 5}}$ it is conceivable, therefore, that injection of epinephrine may cause an acute transient inhibition of insulin release which may at least partly contribute to epinephrine-hyperglycemia by increasing glucose output by the liver.

The complex mechanism by which epinephrine enhances hepatic glucosc output is by no means fully elucidated, and more studies will be required to clarify the situation further. Apparently, however, glucagon is not a mediator for the action of epinephrine on the liver.

\section{ACKNOWLEDGMENT}

The author is grateful to Clifford Reynolds, Mrs. Sandra Jackson, and Miss Phyllis Lumpkin for their technical assistance, and to Mrs. Dorothy Sanbar and Miss Donna Cordon for typing the manuscript.

\section{REFERENCES}

1. Sanbar, S. S., and Reynolds, C.: Effects of epinephrine (E) and glucagon (G) on glucose turnover (abstract). Clin. Res. 14:442, 1966.

2. Sokal, J. E.: Editorial. Glucagon-an essential hormone. Amer. J. Med. 41:331, 1966.

3. Sherlock, S.: Comparison of the carbohydrate effects of adrenalin infused into the femoral vein, carotid artery, aorta and portal vein of rats. Amer. J. Physiol. 157:52, 1949.

4. Ezdinli, E. Z., and Sokal, J. E.: Comparison of glucagon and epinephrine effects in the dog. Endocrinology 78:47, 1966.

5. Sokal, J. E., Sarcione, E. J., and Henderson, A. M.: Relative potency of glucagon and epinephrine as hepatic glycogenolytic agents. Studies with the isolated per- fused rat liver. Endocrinology 74:930, 1964.

6. Hetenyi, G., Jr., Rappaport, A. M., and Wrenshall, G. A.: Effects of insulin on the tracer-determined distribution and production of glucose in liverless dogs. Diabetes 12: $150,1963$.

7. Steele, R.: Influences of glucose loading and of injected insulin on hepatic glucose output. Ann. N. Y. Acad. Sci. 82:420, 1959.

8. Sanbar, S. S.: Effect of L-leucine on glucose turnover in dogs. Metabolism 15: 557, 1966.

9. Eadie, G. S.: A comparison of the glycogenolytic responses to epinephrine administered by the subcutaneous and intravenous routes. Amer. J. Physiol. 90:711, 1929. 
10. Dworkin, S.: The mechanism of adrenalin glycemia. Amer. J. Physiol. 93:646, 1930.

11. Rosenberg, F. J., and DiStefano, V.: A central nervous system component of epinephrine hyperglycemia. Amer. J. Physiol. 203:782, 1962 .

12. Ellis, $S$ : The metabolic effects of epinephrine and related amines. Pharmacol. Rev. 8:485, 1956.

13. De Bodo, R. C., Steele, R., Altszuler, N., Dunn, A., and Bishop, J. S.: On the hormonal regulation of carbohydrate metabolism; studies with $\mathrm{C}^{14}$ glucose. Recent Progr. Hormone Res. 19:445, 1963.

14. Sutherland, E. W., and Rall, T. W.: The relation of adenosine- $3^{\prime}, 5^{\prime}$-phosphate and phosphorylase to the actions of catecholamines and other hormones. Pharmacol. Rev. 12:265, 1960.

15. - , and Cori, C. F.: Effect of hyperglycemic-glycogenolytic factor and epinephrine on liver phosphorylase. J. Biol. Chem. 188:5.31. 1951.

16. Lundsgaard, E., Neilsen, N. A., and Orskov, S. L.: The carbohydrate metabolism of the isolated cat liver. Skand. Arch. Physiol. $73: 296,1936$.

17. - - - and - - : On the possibility of demonstrating an effect of insulin on isolated mammalian liver. Skand. Arch. Physiol. 81:11, 1939.

18. Leimdorfer, A., Arana, R., and Hack, M. H.: Hyperglycemia induced by the action of adrenalin on the central nervous system. Amer. J. Physiol. 150:588, 1947.
19. Sergeyeva, M. A.: Microscopic changes in the islands of Langerhans produced by sympathetic and parasympathetic stimulation in the cat. Anat. Record 77: $297,1940$.

20. Coore, H. G., and Randle, P. J.: Regulation of insulin secretion studied with pieces of rabbit pancreas incubated in vitro. Biochem. J. 9:3:66, 1964

21. Kosoka, K., Ide, T., Kuzuya, T., Miki, E., Kuzuya, H., and Okinaka, S.: Insulin-like activity in pancreatic vein blood after glucose loading and epinephrine hyperglycemia. Endocrinology 75:9, 1964.

22. Porte, D., Jr., Graber, A. L., Kuzuya, $\mathrm{T}$., and Williams, R. H.: The effect of epinephrine on immunoreactive insulin levels in man. J. Clin. Invest. 4:228, 1966.

23. Franckson, J. R. M., Arnould, Y., Malaisse, W., and Conrad, V.: Glucose metabolism in normal anesthetized dog injected successively with anti-insulin serum and insulin. Diabetes 13:532, 1964.

24. Altszuler, N., Steele, R., Tobin, J., Rathgel, I., and de Bodo, R. C.: Effect of anti-insulin serum on glucose production and uptake on dog: (abstract). Fifth Congress of the International Diabetes Federation, Toronto. Excerpta Medica, International Congress Series 74:168, 1964.

25. Wrenshall, G. A., Vranic, M., Cowan, J. S., and Rappaport, A. M.: Effects of Sudden Deprivation and Restoration of Insulin Secretion on Glucose Metabolism in Dogs. Diabetes 14:689, 1965. 